\title{
Chitosan Channels Stuffed with Mesenchyme Originated Stem/Progenitor Cells for Renovate Axonal Regeneration in Complete Spinal Cord Transection
}

\author{
Ahmet Tulgar BASAK ${ }^{1}$, Nazli CAKICl ${ }^{2}$, Gokhan BOZKURT ${ }^{3}$, Nuhan PURALI ${ }^{4}$, Emir Baki DENKBAS ${ }^{5}$, \\ Petek KORKUSUZ ${ }^{6}$, Duygu UCKAN CETINKAYA ${ }^{7}$ \\ ${ }^{1}$ Koc University Hospital, Department of Neurological Surgery, Istanbul, Turkey \\ ${ }^{2}$ Medicana International Hospital, Department of Neurological Surgery, Istanbul, Turkey \\ ${ }^{3}$ Memorial Bahcelievler Hospital, Department of Neurological Surgery, Istanbul, Turkey \\ ${ }^{4}$ Hacettepe University School of Medicine, Department of Biophysics, Ankara, Turkey \\ ${ }^{5}$ Hacettepe University, Department of Nanotechnology, Ankara, Turkey \\ ${ }^{6}$ Hacettepe University School of Medicine, Department of Histology, Ankara, Turkey \\ ${ }^{7}$ Hacettepe University School of Medicine, Department of Stem Cell Research Center, Ankara, Turkey \\ Corresponding author: Nazli CAKICl drnazlicakici@yahoo.com
}

\section{ABSTRACT}

AIM: To examine the implantation of chitosan channels stuffed with mesenchyme-originated stem/progenitor cells (MSPCs) derived from adult rats in a spinal cord transection model. The level of axonal regeneration, the effect of chitosan channels on the survival of MSPCs, and the functional recovery results were also evaluated.

MATERIAL and METHODS: Chitosan channels stuffed with MSPCs were implanted at the level of T8 in a transected rat spinal cord. MSPCs were harvested from the pelvic bone marrow of adult rats, and the MSPC-chitosan channel group was compared with three control groups. The axonal regeneration capacity, the effect of chitosan channels on the survival of MSPCs, and the functional recovery results were compared among four groups. The survival of MSPCs was evaluated using histopathological techniques and electron microscopy, axonal regeneration/germination was evaluated by confocal microscopy, and locomotor function was assessed for 4 weeks using the Basso, Beattie, and Bresnahan locomotor score.

RESULTS: The MSPC-chitosan channel group exhibited enhanced survival of transplanted MSPCs compared with MSPCs transplanted directly into the lesion cavity, although no significant difference was detected in locomotor function between the treatment and control groups. The MSPC-chitosan channel group demonstrated thicker myelination of axons than the other groups. CONCLUSION: Chitosan channels promoted the survival of transplanted MSPCs and created a tissue bridge after complete spinal cord transection. They also induced axonal regeneration and germination. No significant improvement in functional recovery was found between the groups.

KEYWORDS: Mesenchyme-originated stem/progenitor cells, Axonal regeneration, Chitosan channel, Complete spinal cord transection

ABBREVIATIONS: BBB: Basso, Beattie, Bresnahan, BMSPCs: Bone marrow-derived mesenchymal stem/progenitor cells, CNS: Central nervous system, CSF: Cerebrospinal fluid, DiO: 3,3-Dioctadecyloxacarbocyanine perchlorate, DiL: 1-1'-Dioctadecyl3,3,3',3'-Tetramethylindocarbocyanine perchlorate, DMEM: Dulbeco's modified eagle medium, EAE: Experimental aotuimmune encephalomyelitis, G-CSF: Cerebrospinal fluid derived growth factor, GFAP: Glial fibrillary acidic protein, MSPCs: MesenchymeOriginated Stem/Progenitor Cells, NaOH: Sodium hydroxide, NGF: Neural growht factor, NSPCs: Neural stem/progenitor cells, SCT: Spinal cord transection, TEM: Transmission electron microscope

Ahmet Tulgar BASAK (1) : 0000-0002-7282-3079

Nazli CAKICl

Gokhan BOZKURT
(D) : 0000-0003-1480-6235

(D) : 0000-0001-5135-1343
Nuhan PURALI

(1) : $0000-0002-0604-2303$

Emir Baki DENKBAS (D) : 0000-0002-2190-774X

Petek KORKUSUZ (1) : 0000-0002-7553-3915

Duygu UCKAN CETINKAYA (D) : 0000-0003-3593-6493 


\section{- INTRODUCTION}

A Ithough complete spinal cord injury is considered as an incurable condition, several recent studies have demonstrated that recovery after spinal cord injury is possible. The central nervous system has stem cells that are capable of diffentiating into other neuronal cells. Although these cells can differentiate into neuronal tissue, other problems such as the response of the body to acute trauma and the loss of connection between the cut ends of the spinal cord render the regeneration process impossible. A traumatic spinal cord injury results in highly complex pathophysiological responses in the lesion area (24). During this period, neurotransmitters such as glutamate, aspartate, glial fibrillary acidic protein (GFAP), oxygen radicals, and neuromediators such as growth factors, leukotrienes, and cytokines collect in the lesion area (6). Vasospasm, caused due to damage to the vascular structures, triggers local ischemia, hypoxia, and hypoglycemia. All these factors lead to the death of neuronal and glial cells in the lesion area, subsequently resulting in axonal degeneration. This sequence of events consequently leads to the development of reactive gliosis (16). Damage to axons in the CNS primarily results in complete degeneration of the distal axons and partial degeneration and retraction of the proximal axons. In this stage, it is believed that exogenous stem cells can be used to overcome the insufficiency of endogenous response. The literature reports the use of stem cells isolated from different sources to enhance the recovery process (4). Mesenchyme-originated stem/progenitor cells (MSPCs) have been found to be more advantageous than other stem cells because of their various biological properties (1). MSPCs in the bone marrow secrete various growth factors and cytokines required for the survival and differentiation of hematopoietic stem cells (25). Besides the bone marrow, MSPCs are known to be present in a wide variety of organs and tissues, including the adipose tissue, periosteum, synovial membrane, synovial fluid, skeletal muscle, dermis, umbilical cord blood, placenta, liver, spleen, and thymus $(5,22)$. It has been demonstrated that MSPCs can differentiate into several different types of cells, both mesenchymal and nonmesenchymal, including myocytes, cardiomyocytes, hepatocytes, insulin-producing cells, endothelial cells, epithelial cells, epithelium-like cells, and neurons (11). Based on the belief that MSPCs could have a potential role in the treatment of different diseases, there is a growing interest in understanding their biology, and with the increase in knowledge on this issue, further progress can be achieved in the protocols designed for MSPC-based therapies (11). Like other stem cells, MSPCs are also known to migrate to the affected areas and settle in these regions (26). The ability of MSPCs to migrate through the blood-brain barrier is another important feature (25). Therefore, in spinal cord injuries, stem cell transplantation alone can be inadequate for the regeneration process because of, as we have mentioned earlier, the other problem of the loss of connection between the ends of the spinal cord. Hence, it is necessary to provide a "bridge" structure for bringing both the ends of the lesion closer. The bridge structure must be a biodegradable and biocompatible material to avoid an additional injury to the spinal cord and stem cells.
Chitosan is a biopolymer of cellulose that is obtained by the deacetylation of chitin. The chitosan biopolymer, owing to its biocompatibility, biodegradability, nontoxicity, and antifungal and antimicrobial properties, is used in several different applications (10). Another reason for the extended use of chitosan is that it can be easily converted into the desired shape and geometry (membrane, spongy structure, fiber, microcide and nanocide, and tube). Nontoxic chitosan has been used as an artificial bridge for the repair of sciatic nerve injury in rodents and dogs $(13,23)$.

Therefore, in this study, our objective was to evaluate the results of extramedullary implantation of adult rat MSPCs with a biomaterial, chitosan tube, in an experimental spinal cord transection model. We also investigated the effect of this biomaterial on the proliferation, survival, and axonal regeneration of the transplanted stem cells.

\section{MATERIAL and METHODS}

\section{Production of Chitosan Channels}

Chitosan channel preparation process has been described previously in the literature. Briefly, $5 \mathrm{~g}$ medical-grade chitosan (Protosan UP CL 213, NovaMatrix, Brakeroya, Drammen, Norway) was dissolved in $500 \mathrm{ml}$ of distilled water, and this solution was precipitated using $40 \mathrm{ml}$ of $4 \% \mathrm{wt} / \mathrm{wt}$ sodium hydroxide $(\mathrm{NaOH})$ solution for 24 hours. The remaining preparation process was implemented according to the literature protocols until the chitosan tube formation stage $(10,19)$. Tubular forming of chitosan was achieved with some changes (4). The tubes were $10 \mathrm{~mm}$ in length, $4.1 \mathrm{~mm}$ in outer diameter, and the wall thickness was $0.21 \mathrm{~mm}$ (Figure 1A). All tubes were sterilized by ethylene oxide gas. Next, the tetrazolium test was performed to measure cytotoxicity in chitosan channels. The solutions that interacted with the sample groups in this medium were compared with the media (control group) that did not interact with any sample group. Cell viability in the tested samples was evaluated by the MTT assay at the end of 24-hour cell proliferation. Based on the results, the chitosan concentrations of $5 \%, 6 \%$, and $7 \%$ were not found to have any cytotoxic effect compared with the control group (Figure 2).

\section{Isolation and Culture of MSPCs}

All MSPCs were isolated from $2 \mathrm{ml}$ of pelvic bone marrow obtained from healthy adult Wistar Hannover female rats using the "Ficoll Extraction" sort-out method $(2,15)$. To increase the number of MSPCs, they were allowed to reproduce in an incubator (at $37^{\circ} \mathrm{C}$ and $5 \% \mathrm{CO}_{2}$ ) in Dulbecco's modified Eagle medium (DMEM) supplemented with L-glutamine and fetal calf serum. Trypsin-EDTA enzyme was applied to the cells grown in the culture, and the cells were removed from the culture dish surface. Cells in suspension were stained with trypan blue and counted on Thoma slides. Morphological characterization of cells was performed using an inverted microscope. Next, MSPC-positive (CD105, CD44, and CD73) and -negative (CD45, CD34, and HLA-DR) surface markers and immunophenotype properties were determined using the FACS Bee device. Furthermore, these cells were 

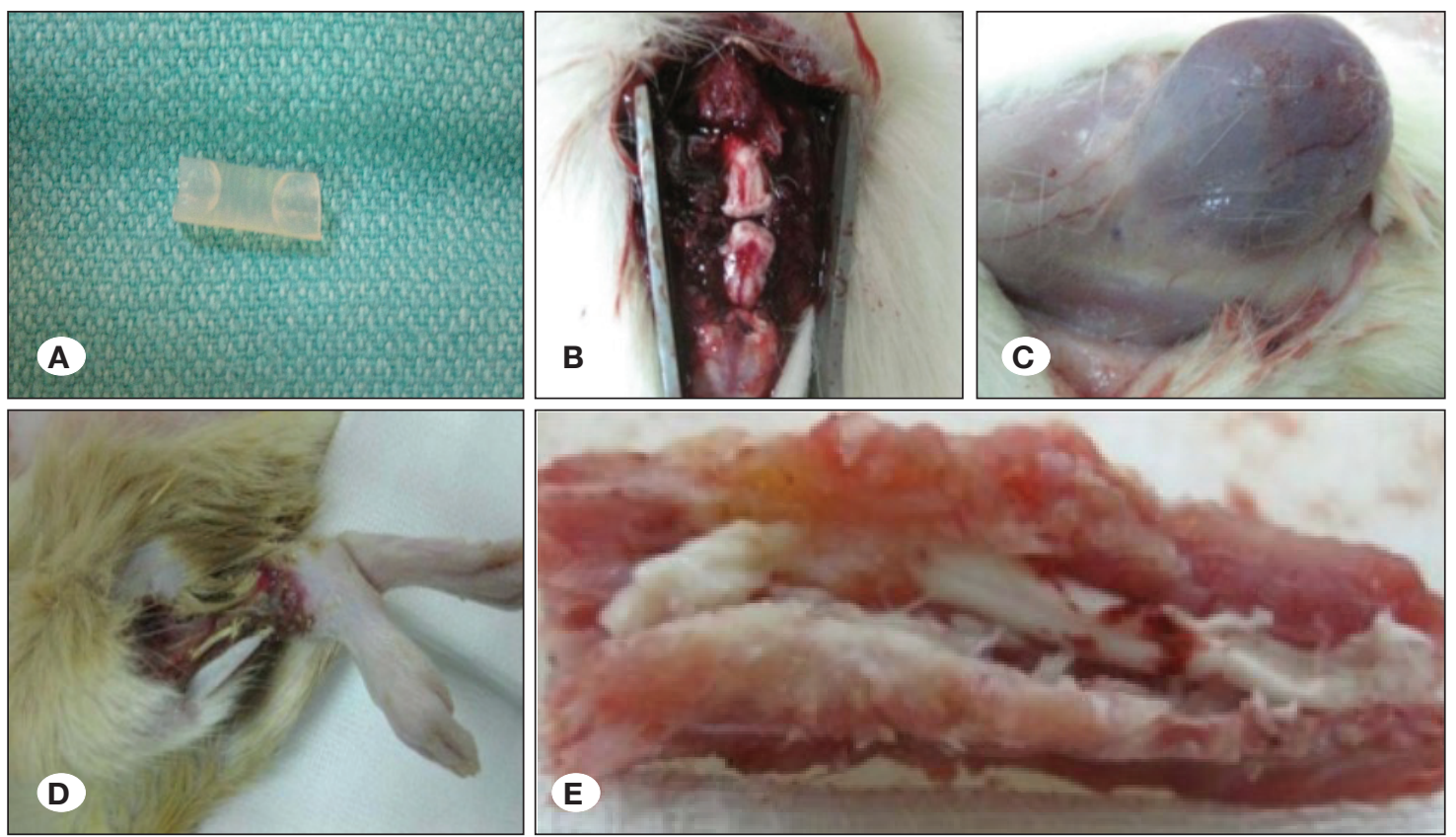

Figure 1: A) Stem cell constructed chitosan tube. B) Surgical microscopic view of the, spinal cord transection.

C) Development of pseudomeningocele. D) Otophagia phemonenon. E) Transected spinal cord tissue in the vertebral column.

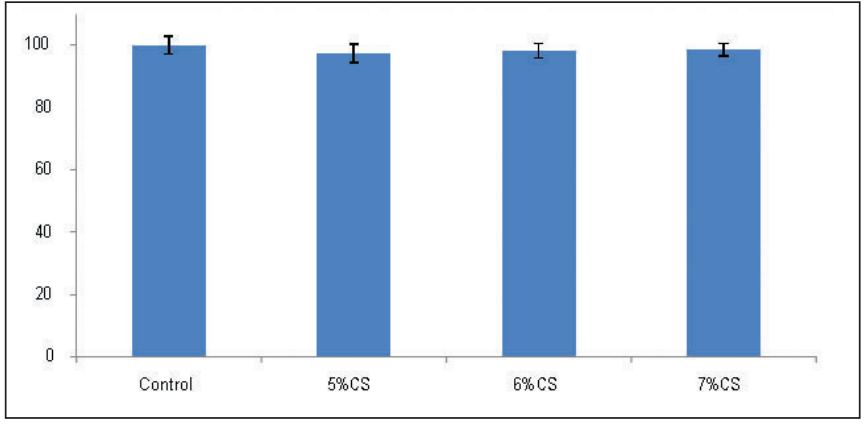

Figure 2: Chitosan channel MTT results.

differentiated into fat, bone, and cartilage cells to ensure their differentiation potentials. MSPCs were stained with the 3,3-dioctadecyloxacarbocyanine perchlorate (DiO) fluorescent dye. The labeled cells were incubated at $37^{\circ} \mathrm{C}$ for $10 \mathrm{~min}$ before administration to the animals. The MSPCs were stuffed into the chitosan channels at a density of $0.5 \times 10^{6} / 10 \mathrm{ml}$ of complete medium.

\section{Animals}

A total of 32 adult female Wistar Hannover female rats weighing 225-275 g were used in this study. The Animal Care Committee of the Research Institute of the Hacettepe University Health Network approved the animal protocols that were in accordance with the policies established by the Republic of Turkey Animal Protection Law. The experimental protocol of the study was examined and approved by the Ethics Committee of Experimental Animals of Hacettepe University with the decision number 2012/32-04.

Rats with SCT were divided into four equal groups as follows: the chitosan channel+MSPC group (CK group) had complete SCT and implantation of chitosan channels stuffed with
MSPCs ( $\mathrm{n}=8$ the empty chitosan channel group ( $\mathrm{C}$ group) had complete SCT and implantation of chitosan channels without MSPCs ( $n=8)$; the MSPC group (K group) had complete SCT and implantation of MSPCs without chitosan channel $(n=8)$; and the transection alone group (T group) had complete SCT alone $(n=8)$. All animals had spinal instrumentation. During the experiment, the animals were maintained under standard laboratory conditions and fed with standard feed.

\section{Spinal Cord Transection and MSPC Implantation}

Each of the 32 rats was anesthetized with ketamine $(50 \mathrm{mg} /$ $\mathrm{kg}$ ) and xylasine hydrochloride $(2 \mathrm{mg} / \mathrm{kg}$ ) via intraperitoneal injection. Preoperatively, $5 \mathrm{mg}$ gentamicin was administered. Surgical procedures were performed using the Zeiss Opmi99 laboratory microscope and microsurgical instruments. After sterile coverage, a skin incision and bilateral paravertebral muscle dissections, and T7, T8, and T9 total laminectomies were performed. At the level of the T8 laminae, the spinal cord was completely transected (Figure 1B). Axonal marking was performed four times in each rat using 1-1'-dioctadecyl3,3,3',3'-tetramethylindocarbocyanine perchlorate (DiL) fluorescent dye using a fine needle. In the CK group, chitosan channels were seeded with $10 \mathrm{ml}$ of medium containing $0.5 \times$ $10^{6}$ MSPCs 10 min before intramedullary transplantation. For the direct injection of MSPCs without chitosan channels (K group), $10 \mathrm{ml}$ of MSPCs was injected into the injury cavity using a Hamilton syringe. The $\mathrm{C}$ group received a similar operative procedure and was transplanted with only chitosan channels. The T group had complete SCT without implantation of chitosan channels or MSPCs. After this procedure, spinal fixation was performed using a surgical steel wire according to the model defined by Nomura et al. (19). To aid transplant survival, all animals were immunosuppressed daily using cyclosporine. Bladders were evacuated three times daily. The rats were housed separately in a temperature-controlled 
room at $26^{\circ} \mathrm{C}$ with a 12 -hour light/dark cycle. Water and food were provided ad libitum. Three blinded observers analyzed the locomotor recovery two times weekly during the 4-week survival period using the Basso, Beattie, and Bresnahan (BBB) open field locomotor test, and all animals were scored for 4 minutes two times every week.

\section{Anterograde Axonal Tracing with DiO and DiL}

In transplantation studies, it is necessary to observe whether the transplanted cells adhere to the recipient tissue and survive there. DiL and DiO membrane marker dyes are used for stem cell marking as they are highly fluorescent and render the cells to be recognized. Using confocal microscope, we examined the MSPCs with DiO-labeled (green stigma) and DiL-labeled (red stigma) axons in all our study groups.

\section{Tissue Preparation and Histochemistry}

After 4 weeks, the rats were perfused with $4 \%$ paraformaldehyde $(0.1 \mathrm{~mol} / \mathrm{l}$ in phosphate buffer, $\mathrm{pH}$ 7.4) from the left ventricle at a constant velocity until the extremities of the animal were hardened. A 3-cm segment of the spinal cord of the injury site was removed and fixed by immersion in glutaraldehyde solution at $40^{\circ} \mathrm{C}$ for $4-6 \mathrm{~h}$. It was then washed in phosphate buffer solution and post-trapped in phosphate-buffered $1 \%$ osmium tetroxide at $4^{\circ} \mathrm{C}$ for $2 \mathrm{~h}$. Tissue samples were passed through graded ethyl alcohol series by a tissue tracking device with constant agitation (Leica Wetzlar, Germany) and and embedded in Epone (EMS, Germany). Semi-thin sections (at least 10 sections per sample) were analyzed by computed light microscopy and a digital camera (Leica DC500, Germany). The tissue response to the implant (biomaterial) was scored semiquantitatively according to soft tissue implants. The tissue response of the outer face of the tube could not be evaluated as the chitosan tube was removed before the samples were subjected to histological examination.

\section{Transmission Electron Microscopy}

One animal from each of the CK, K, C, and T groups was randomly selected for transmission electron microscopy (TEM). Thin sections were stained with uranyl acetate-lead citrate in an automatic staining device (Leica, Germany) and evaluated using an Orius digital camera connected to a Jeol JEM-1400 model TEM and in terms of the morphological repair criteria of the spinal cord. For this purpose, at least 10 axon thicknesses per at least three thin sections of each sample were measured and recorded in micrometers by TEM.

\section{Transmission Confocal Microscopy}

Cryoprotection was performed to specimens for $6-8 \mathrm{~h}$ in a $30 \%$ sucrose solution. Samples were frozen in liquid nitrogen at $-196^{\circ} \mathrm{C}$, and 40 - to $60-\mu \mathrm{m}$-thick serial sections were cut in a longitudinal plane using a cryostat. For immunohistochemical examination of the areas where axons and stem cells were observed under the confocal microscope, 5- to 7- $\mu$ m-thick sections were fixed in cold acetone for $10 \mathrm{~min}$. Rapidly dried sections were stored at $-30^{\circ} \mathrm{C}$. Under confocal microscopy, the DiO-labeled mesenchymal stem cells (green stigma) and the DiL-labeled axons (red stigma) were applied to each study group.

\section{Behavioral Analysis}

For behavioral analysis, three independent observers analyzed the hindlimb motor function and functional recovery two times a week for 4 weeks after SCT using the BBB open-field locomotor test (3). The score of each hindlimb was recorded, and the median value was presented.

\section{Statistical Analysis}

Data were analyzed at the Department of Biostatistics, Hacettepe University. Independent variables were the groups, and dependent variables were histology and gait analysis measurements. The Shapiro-Wilk test was used to determine whether the data were normally distributed. The KruskalWallis test was used for multiple comparison and MannWhitney test used for paired comparison. Spearman's test was used for correlation analysis, and the Friedman test was done for time change analysis. All data were represented as median, minimum, and maximum values. The analyses were performed using SPSS version 15.0, with the difference being considered to be statistically significant at $p<0.05$.

\section{Ethics}

The Animal Care Committee of the Research Institute of the Hacettepe University Health Network approved the animal protocols in accordance with policies established by the Republic of Turkey Animal Protection Law. The experimental protocol of the study was examined by Hacettepe University Ethics Committee of Experimental Animals and approved by the decision number 2012 /32-04.

\section{RESULTS}

All rats in all groups had uncomplicated wound healing. There was no cerebrospinal fluid fistula in the rats; one rat had pseudomeningocele at the lesion site, and another exhibited autophagy in the right hindlimb (Figure 1C, D).

\section{General Histological Appearance}

Under the light microscope, it was observed that the chitosan channels caused a moderate tissue reaction (Figure 1E), but this reaction was not significantly different between treated and untreated cells. On the inner surface of the channel, a medium-thick (1- to 10-fold) capsule formed with mononuclear phagocytic cells, giant cells, lymphocytes, and fibroblasts. This tissue was consistent with the granulation tissue in which the medial splenic vein-rich medulla spinalis was involved in transection and regeneration. Encapsulation on the outer surface of the tube could not be observed because the tube was removed after sacrifice. The transection area was covered with granulation tissue containing cells and veinrich, loose glial fibrils, necrotic cell debris, and macrophages in all groups. Axons were regenerated at varying degrees in the incision area in all groups. Irregular and different-sized axons were accompanied by a rich capillary network in these areas. The MSPC group (K group) and the MSPC-chitosan channel group (CK group) had more axons per unit area than the other groups. However, due to the small number of rats in each group, no statistically significant increase in the number 
of axons and blood vessels (Figure 3, Table 1). Therefore, mesenchymal stem cell application was found to accelerate the spinal cord repair process slightly according to the tube application alone.

\section{Electron Microscopy}

Electron microscopy demonstrated the incision site and the adjacent gray and white matter, edema, and neuronal and axonal ultrastructural damage. The axons exhibited relaxation and thinning, myelin, separation, swelling, peeling, and damage to the axoplasmic mitochondria. Myelin thickness measurements in the MSPC-chitosan channel group (CK group) were significantly higher than those in the control groups $(p<0.05)$ (Figure 4, 5).

\section{Locomotor Recovery}

BBB scores showed no significant correlation with histological measurements. There were also no significant changes in BBB scores over time between the groups. However, a significant correlation was observed in all groups, except for the 3rd week to the 1st month, when the intragroup days were taken as a basis $(p<0.05)$ (Figure 6).

\section{Confocal Microscopy}

Under the confocal microscope, it was observed that mesenchymal stem cell application accelerated the spinal cord repair process slightly, and the myelin sheath repair process was higher in the stem cell and stem cell-chitosan tube groups than in the control group. Myelin thickness

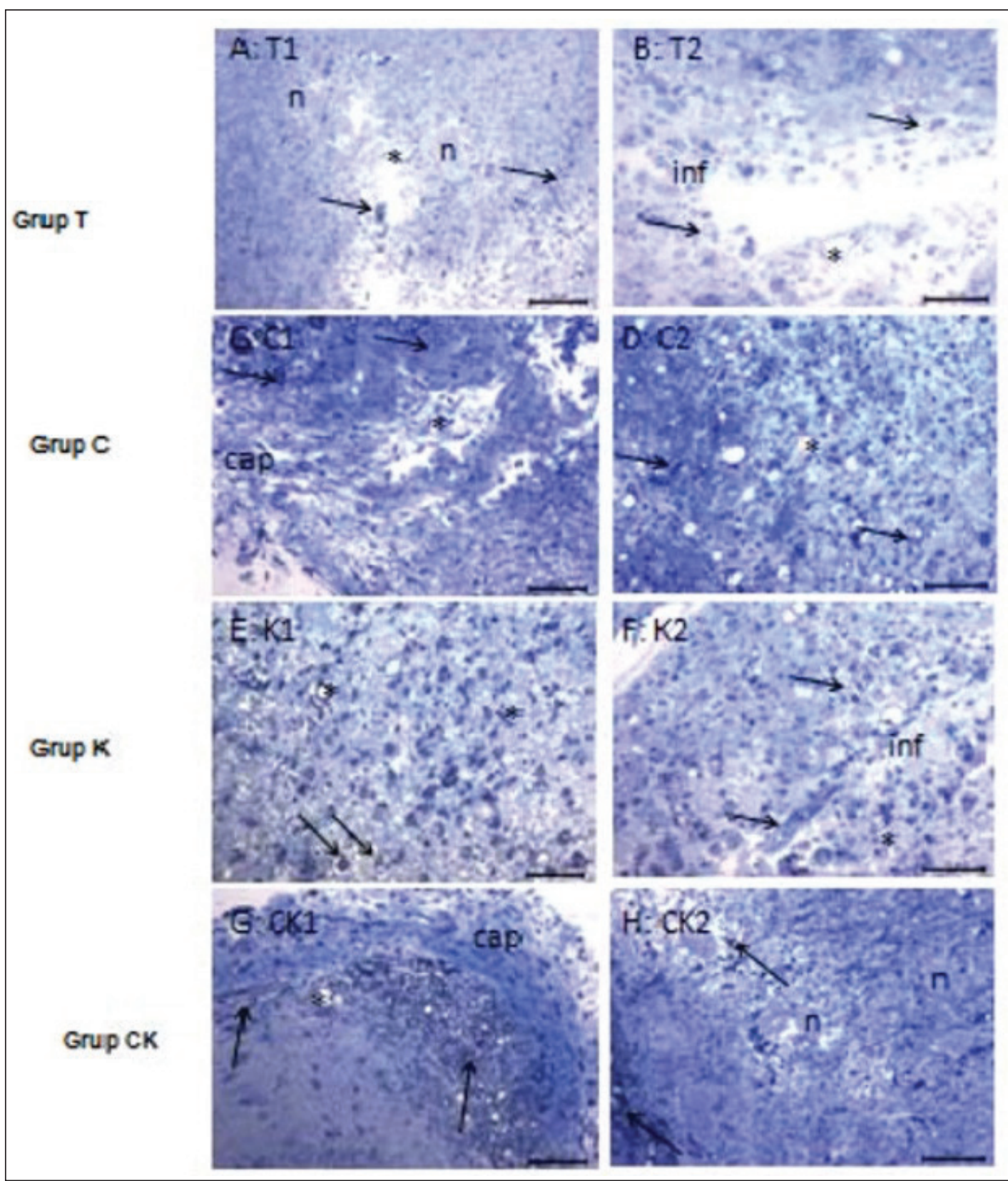

Figure 3: The

micrographs of all groups of the transverse medulla spinalis semi-thin sections showing the granulation tissue containing necrotic cell debris, which is rich in vein (asterisk) and inflammatory cells (inf) at the incision site. It is observed that the granulation tissue is more organized in the group of stem cells than in the other groups. The fibrous encapsulation surrounding the chitosan tube is shown in $C$ and $G$. $\mathrm{N}$ : Neuron body,

Arrow: Axon,

Asterisk: Vein, Inf: Infiltration of inflammatory cells. K: Capsule. Methylene blue-Azur II $\times 400$. 
Table I: Biomaterial Tissue Response Scale (Internal Part of the Chitosan Channel in the Response Tissue. Biomaterial Response Microscopic Properties of the Enveloping Capsule are Scored. Result Obtained for Each Sample Total Score is Recorded)

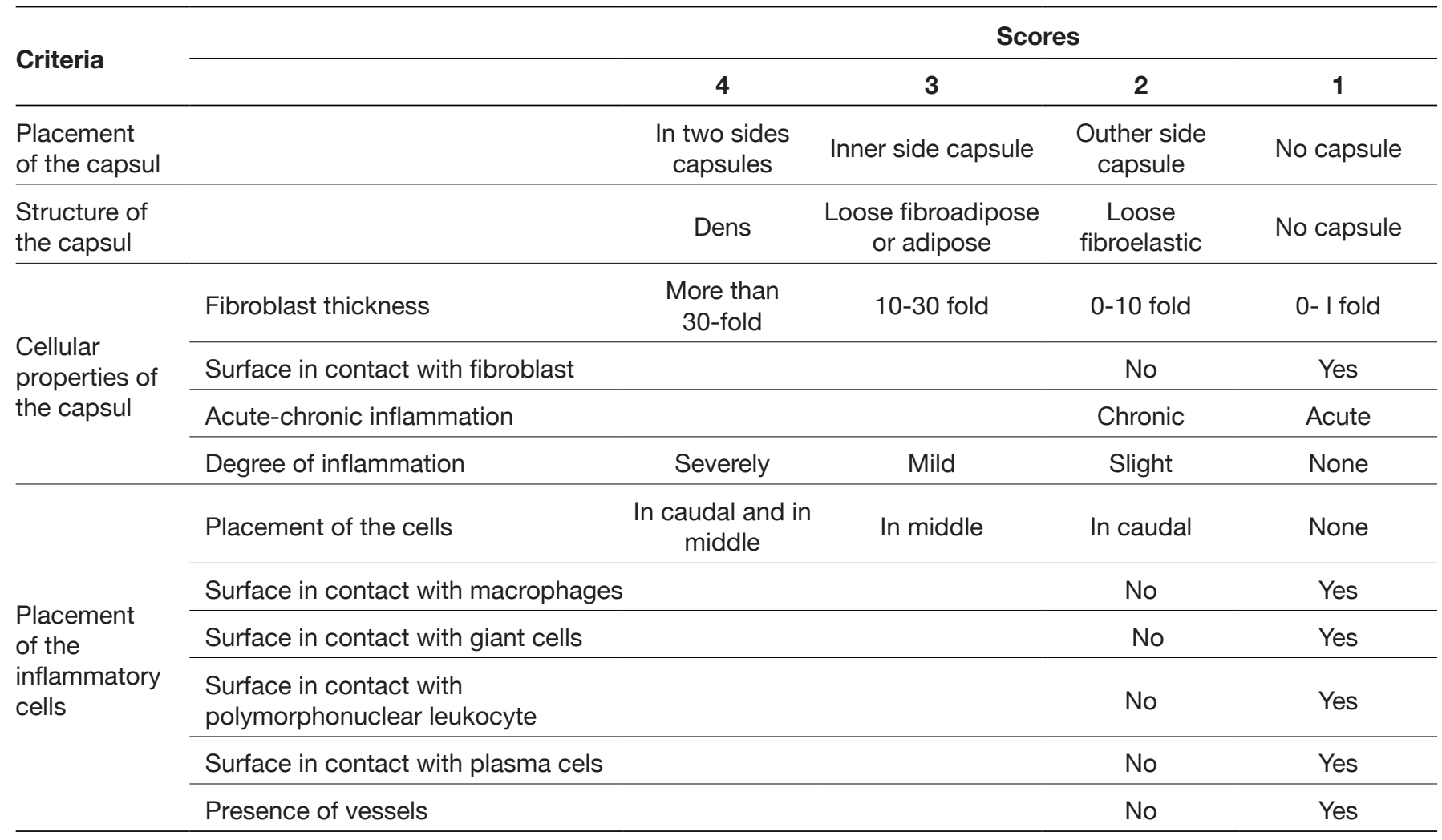

measurements in these groups were significantly higher than those in the control group $(p<0.05)$ (Figure $7 A-D)$.

\section{DISCUSSION}

The primary strategy in the treatment of spinal cord injuries is to increase the endogenous response of the spinal cord toward the neurotrophic direction. After $\mathrm{SCl}$, the secondary injury response results in the formation of a cystic cavity surrounded by an astrocytic scar and an unsuitable microenvironment for regeneration. For this purpose, various bridging biomaterials, cell or genomic transfers, various enzymes or growth factors, and/or a combination of these factors are attempted. Approximately in last 25 years, studies were focused on the results of the combination of bridging biomaterials/polymers and stem/progenitor cells procured from different origins.

Bone marrow-derived mesenchymal stem/progenitor cells (BMSPCs) have potentials to differentiate into mesodermal, neuronal, or glial cells in experimental models $(9,10)$. MSPCs have significant antiproliferative, anti-inflammatory, and antiapoptotic features that enhance their therapeutic potentials (9). In experimental models of autoimmune encephalomyelitis $(E A E)$, brain ischemia, and brain or spinal cord injury, the therapeutic properties of MSPCs have been found to be highly effective $(9,23)$. Some researchers have reported that MSPCs have immunosuppressive properties and due to their regenerative potential, BMSPCs can reduce inflammation in the trauma area, cavity formation, and astrocyte and microglial reactivity (12). Moreover, BMSPC transplantation was found to decrease the hypersensitivity reaction in the trauma area and increase the neuronal and cellular protection in an experimental $\mathrm{SCl}$ model (9).

Several agents such as growth factors, immunosuppressive factors, and bridge-forming structures are used to enhance the viability of transplanted NSPCs $(4,18,20)$. The rationale for using these factors includes the provision of a route for guided axonal regeneration and decreasing the barrier caused by posttraumatic scar tissue. Moreover, it involves the production of neurotrophic factors to enhance endogenous cell survival and axonal regeneration and delivery of cellular and noncellular agents to limit the secondary injury response. In the tissue, bioengineering technique may also enhance the survival or differentiation of stem/progenitor cells (4) $(17,19)$. Several types of biomaterials and devices produced by tissue engineering systems have been used as scaffolds, channels, or delivery systems to enhance neuroprotection and neuroregeneration in experimental $\mathrm{SCl}$ models $(4,18)$. In our study, light microscopy revealed that the chitosan tube caused a moderate tissue reaction. The resulting tissue reaction was significantly different between the treated and untreated cells. Encapsulation on the outer surface of the tube could not be observed because the tube was removed after sacrifice. Under the confocal microscope, the meso-stem cells containing the DiO-labeled mesenchymal stem cells (green stigma) and the DiL-labeled axons (red stigma) were applied to each group. A portion of the fluorescently labeled cells was found to be 


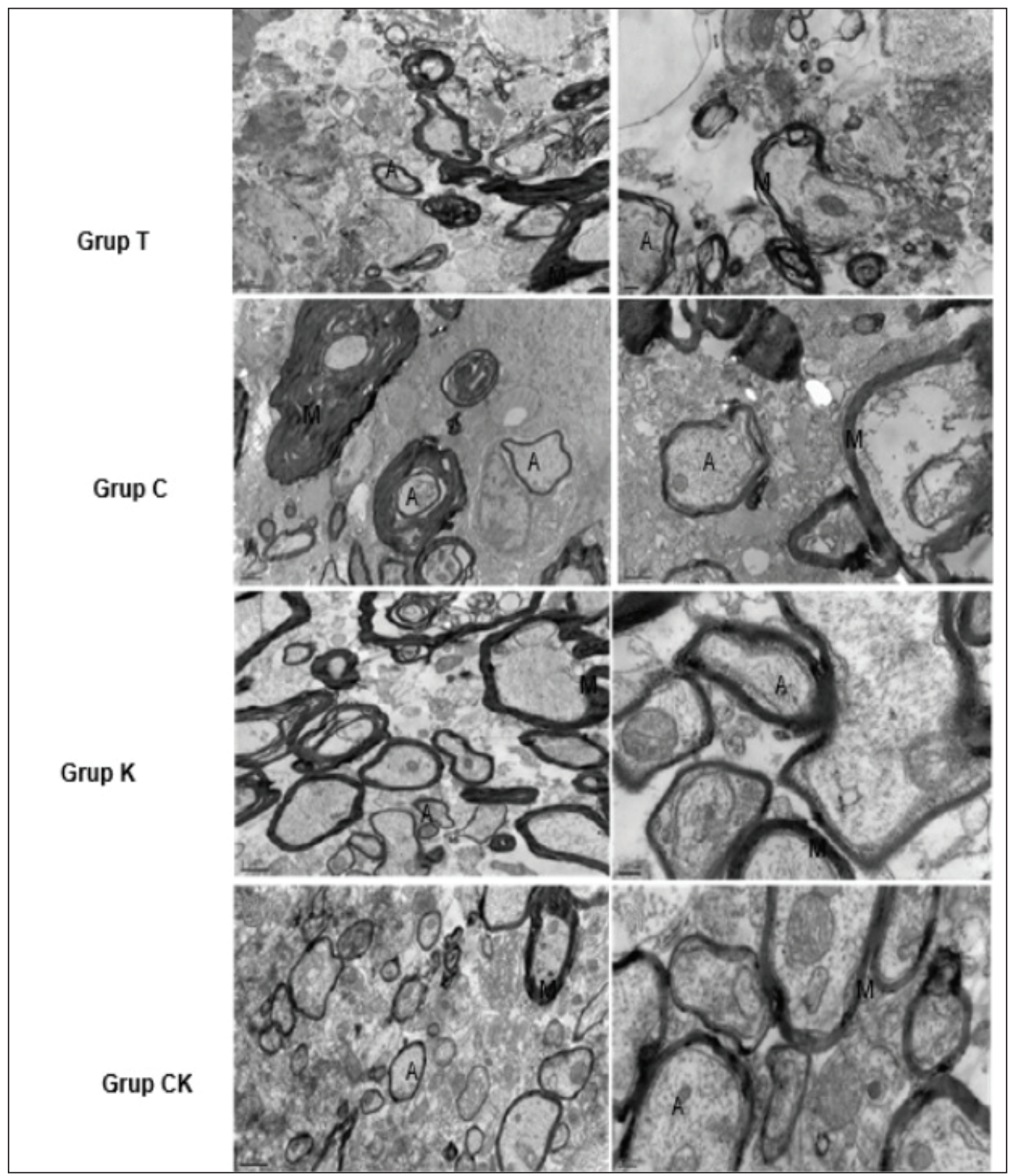

Figure 4: Cross-sectional area and electronmicrographs of the neighboring repair area. Ultrastructural damage (myelin rupture, melting, swelling, thinning, detachment) is observed in the left column in small $(\times 12,000$ or $\times 150,000)$ and larger $(\times 40,000$ or $\times 50,000)$ magnification.

Degenerative changes in groups of stem cell and stem cell-chitosan tubes are observed to be more advanced in the repair process by remyelination of axons. A: Axon; M: Myelin. Uranyl acetate-lead citrate.

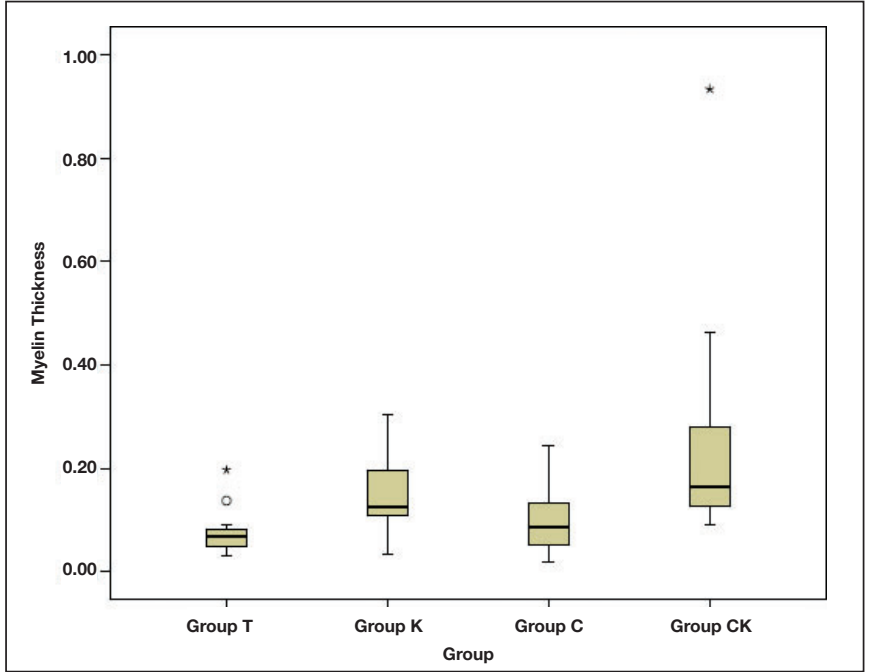

Figure 5: Myelin thickness per group.

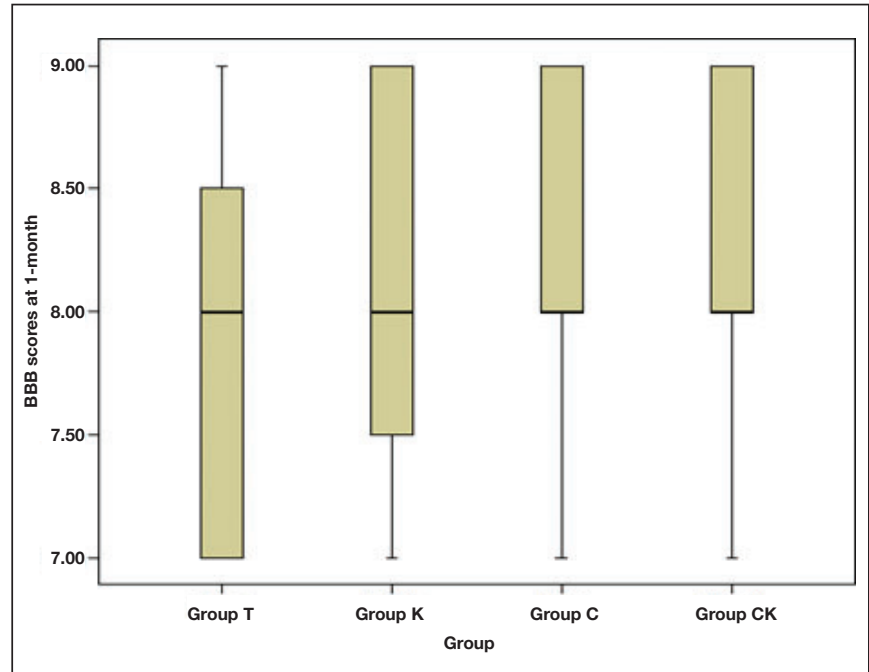

Figure 6: BBB scores at 1-month. 

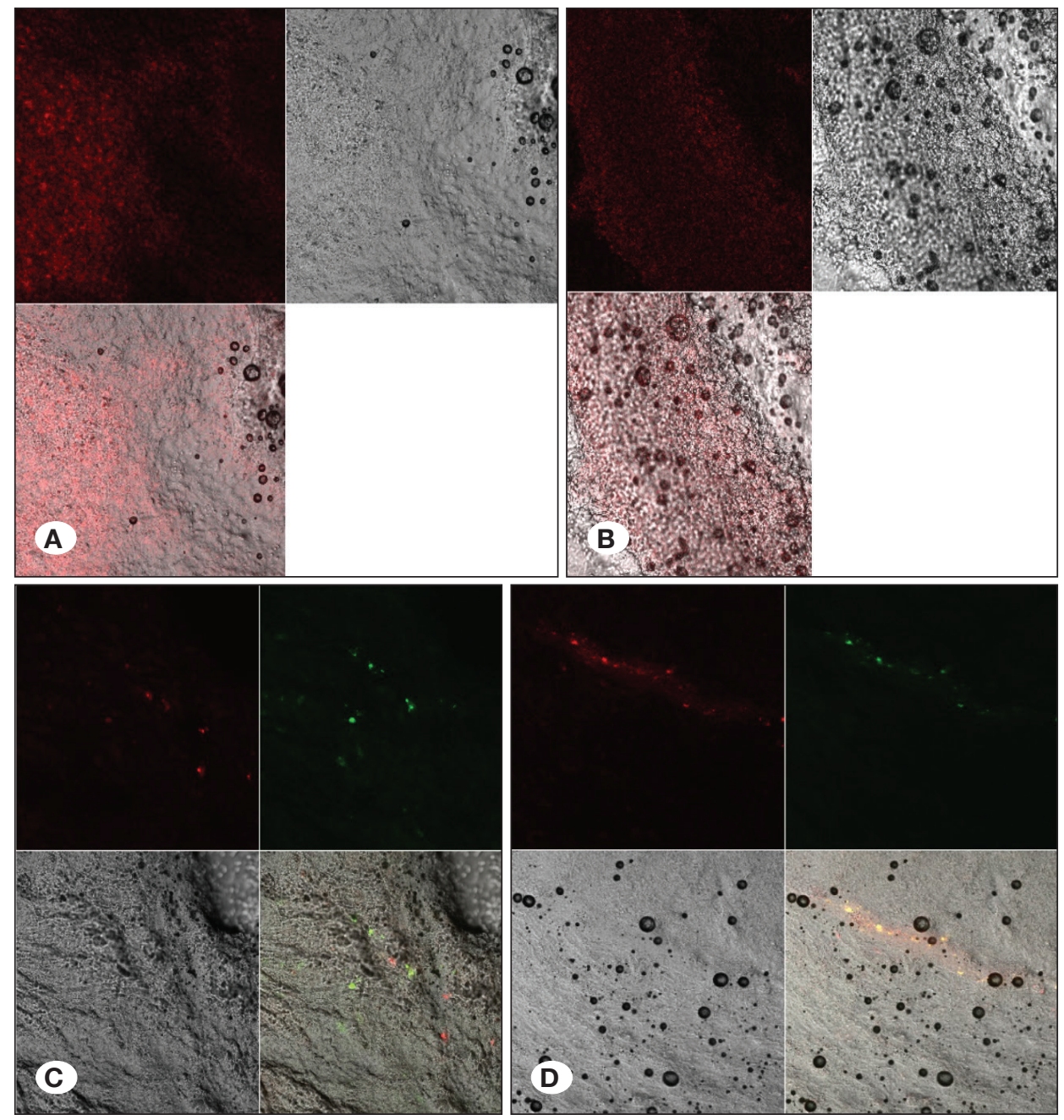

Figure 7: A) Images of axons in Group $\mathrm{C}$ with tongue fluorescent dye. On the right side, respectively, purple fluorescent, Tmldm mode and superposed cases of both images. B) Images of axons in Group T with marked fluorescent dye. On the right side, respectively, purple fluorescent, Tmldm mode and superposed cases of both images.

C) DiO fluorescent marked images of tongue fluorescent dyeing and transplanted mkhs of axons in Group K. Right fluid fluorescent language and DiO images, simulated mode and superimposed cases of both images. D) DiO fluorescent marked images of tongue fluorescent dyeing and transplanted mkhs of axons in Group C. Right fluid fluorescent language and DiO images, simulated mode and superimposed cases of both images. scattered at the lesion site. We observed that mesenchymal stem cell application accelerated the spinal cord repair process slightly and that the myelin sheath repair process was higher in the stem cell and stem cell-chitosan tube groups than in the control group. Myelin thickness measurements in these groups were significantly higher than those in the control group $(p<0.05)$ (Figure 7A, 4B-D). Regarding the effects of MSPCs administered with various polymers on locomotor development in rats reported in the literature, we found six studies that reported functional improvement. However, these studies had a few issues. For example, repetitive targeted IT transplantation of MSPCs at an optimal dosage can improve functional recovery $(7,9)$. Cell delivery via 3D scaffolds was feasible especially in incomplete spinal cord transection (9) (14). The other issues were the different action mechanisms of MSPCs, BMSPCs, or G-CSF (cerebrospinal fluid) in stimulating functional and morphological recovery, intrinsic properties of MSPCs, microenvironment of the injured spinal cord, and graft vs host reactions $(8,9,21)$.

In our study, BBB scores showed no significant correlation with histological measurements. The BBB scores also showed no significant changes over time between the groups. However, there was a significant correlation in all groups, except for the 3rd week to the 1st month, when the intragroup days were taken as a basis $(p<0.05)$.

\section{CONCLUSION}

We investigated the combined application of chitosan, a biomaterial, and human adult mesenchymal stem/progenitor cells to a trauma model of acute spinal cord injury (transection model). The literature reports several studies conducted using chitosan in the spinal cord transection model as a neuronal stem cell (in allograft or xenograft) $(4,19)$. However, to our knowledge, our study is the first report of the implantation of extramedullary chitosan channels seeded with MSPCs derived from the adult rat pelvic bone marrow after complete SCT, the most severe form of experimental spinal cord injury.

We examined the effect of transplanted mesenchymal stem cells in chitosan tubes in the experimental spinal cord transection model. The combined application of cellular transplantation and bridge-forming biomaterials was conducted to promote the regeneration of the damaged CNS. The functional levels of the rats that survived 4 weeks after the trauma were determined by BBB scoring, and no significant difference was found between the groups. A significant correlation was 
observed in the intragroup time intervals. Histological examinations by electron microscopy showed that the thickness of axonal myelin correlated significantly in favor of the CK group. The light microscopy data were also consistent with this finding. In the evaluations made under confocal microscopy, the meso-chemically induced stem cells were screened in the cross-sectional area of the preparations obtained from the respective groups by incubating with $\mathrm{DiO}$ fluorescent dye and showing the axons marked with cross-sectional areas of DiL fluorescent dye.

Therapeutic application of MSPCs represents a promising approach in the treatment of spinal cord injury.

Stem cell transplantation studies provide a novel perspective on the treatment of spinal cord injuries. However, it is clear that it would take a long time to reach the ideal treatment method. Till date, the pathophysiology of spinal cord injuries has not been completely elucidated. Understanding the processes of degeneration and regeneration and identifying the ideal methods for ideal biomaterials and stem cell transplantation will be the basis for advances in this direction. Till date, MSPCs have been used in several clinical trials for the treatment of $\mathrm{SCl}$, but their therapeutic effects need to be demonstrated by controlled, randomized, double-blind, and long-term clinical trials.

\section{ACKNOWLEDGEMENT}

This study was funded by Hacettepe University, Ankara, Turkey.

The authors would like to thank biologists Damla Turkay, Sevil Arslan and Irem Akar Soycan for excellent technical assistance and helpful discussions.

The authors would like to thank Enago (www.enago.com) for English language review.

\section{REFERENCES}

1. Abdallah BM, Kassem M: The use of mesenchymal (skeletal) stem cells for treatment of degenerative diseases: Current status and future perspectives. J Cell Physiol 218:9-12, 2009

2. Barry FP, Murphy JM: Mesenchymal stem cells: Clinical applications and biological characterization. Int J Biochem Cell Biol 36:568-584, 2004

3. Basso DM, Beattie MS, Bresnahan JC: A sensitive and reliable locomotor rating scale for open field testing in rats. $J$ Neurotrauma 12:1-21, 1995

4. Bozkurt G, Mothe AJ, Zahir T, Kim H, Shoichet MS, Tator $\mathrm{CH}$ : Chitosan channels containing spinal cord-derived stem/ progenitor cells for repair of subacute spinal cord injury in the rat. Neurosurgery 67:1733-1744, 2010

5. Chen Y, Shao JZ, Xiang LX, Dong XJ, Zhang GR: Mesenchymal stem cells: A promising candidate in regenerative medicine. Int J Biochem Cell Biol 40:815-820, 2008

6. Chuong CM, Chen HM: Enhanced expression of neural cell adhesion molecules and tenascin (cytotactin) during wound healing. Am J Pathol 138:427-440, 1991
7. Cizkova D, Novotna I, Slovinska L, Vanicky I, Jergova S, Rosocha J, Radonak J: Repetitive intrathecal catheter delivery of bone marrow mesenchymal stromal cells improves functional recovery in a rat model of contusive spinal cord injury. J Neurotrauma 28(9):1951-1961, 2011

8. Dasari VR, Spomar DG, Cady C, Gujrati M, Rao JS, Dinh DH: Mesenchymal stem cells from rat bone marrow downregulate caspase-3-mediated apoptotic pathway after spinal cord injury in rats. Neurochem Res 32:2080-2093, 2007

9. Dasari VR, Veeravalli KK, Dinh DH: Mesenchymal stem cells in the treatment of spinal cord injuries: A review. World J Stem Cells 6:120-133, 2014

10. Freier T, Montenegro R, Shan Koh H, Shoichet MS: Chitinbased tubes for tissue engineering in the nervous system. Biomaterials 26:4624-4632, 2005

11. Giordano A, Galderisi U, Marino IR: From the laboratory bench to the patient's bedside: An update on clinical trials with mesenchymal stem cells. J Cell Physiol 211:27-35, 2007

12. Houle JD, Tessler A: Repair of chronic spinal cord injury. Exp Neurol 182:247-260, 2003

13. Itoh S, Suzuki M, Yamaguchi I, Takakuda K, Kobayashi H, Shinomiya K, Tanaka J: Development of a nerve scaffold using a tendon chitosan tube. Artif Organs 27:1079-1088, 2003

14. Kang KN, Lee JY, Kim DY, Lee BN, Ahn HH, Lee B, Khang G, Park SR, Min BH, Kim JH, Lee HB, Kim MS: Regeneration of completely transected spinal cord using scaffold of poly(D,Llactide-co-glycolide)/small intestinal submucosa seeded with rat bone marrow stem cells. Tissue Eng Part A 17:2143-2152, 2011

15. Mareschi K, Ferrero I, Rustichelli D, Aschero S, Gammaitoni L, Aglietta M, Madon E, Fagioli F: Expansion of mesenchymal stem cells isolated from pediatric and adult donor bone marrow. J Cell Biochem 97:744-754, 2006

16. Maxwell WL, Follows R, Ashhurst DE, Berry M: The response of the cerebral hemisphere of the rat to injury. I. The mature rat. Philos Trans R Soc Lond B Biol Sci 328:479-500, 1990

17. Nomura H, Baladie B, Katayama Y, Morshead CM, Shoichet MS, Tator $\mathrm{CH}$ : Delayed implantation of intramedullary chitosan channels containing nerve grafts promotes extensive axonal regeneration after spinal cord injury. Neurosurgery 63:127141; discussion 141-143, 2008

18. Nomura H, Tator $\mathrm{CH}$, Shoichet MS: Bioengineered strategies for spinal cord repair. J Neurotrauma 23:496-507, 2006

19. Nomura H, Zahir T, Kim H, Katayama Y, Kulbatski I, Morshead $\mathrm{CM}$, Shoichet MS, Tator $\mathrm{CH}$ : Extramedullary chitosan channels promote survival of transplanted neural stem and progenitor cells and create a tissue bridge after complete spinal cord transection. Tissue Eng Part A 14:649-665, 2008

20. Novikova LN, Novikov LN, Kellerth JO: Biopolymers and biodegradable smart implants for tissue regeneration after spinal cord injury. Curr Opin Neurol 16:711-715, 2003

21. Ohta M, Suzuki Y, Noda T, Ejiri Y, Dezawa M, Kataoka K, Chou $\mathrm{H}$, Ishikawa N, Matsumoto N, Iwashita Y, Mizuta E, Kuno S, Ide C: Bone marrow stromal cells infused into the cerebrospinal fluid promote functional recovery of the injured rat spinal cord with reduced cavity formation. Exp Neurol 187:266-278, 2004 
22. Patel SA, Sherman L, Munoz J, Rameshwar P: Immunological properties of mesenchymal stem cells and clinical implications. Arch Immunol Ther Exp (Warsz) 56:1-8, 2008

23. Rosales-Cortes M, Peregrina-Sandoval J, Bañuelos-Pineda J, Castellanos-Martínez EE, Gómez-Pinedo UA, AlbarránRodríguez E. Regeneration of the axotomised sciatic nerve in dogs using the tubulisation technique with Chitosan biomaterial preloaded with progesterone. Rev Neurol 36:11371141,2003
24. Tymianski $\mathrm{M}$, Tator $\mathrm{CH}$ : Normal and abnormal calcium homeostasis in neurons: A basis for the pathophysiology of traumatic and ischemic central nervous system injury. Neurosurgery 38:1176-1195, 1996

25. Zietlow R, Lane EL, Dunnett SB, Rosser AE: Human stem cells for CNS repair. Cell Tissue Res 331:301-322, 2008 